\title{
A novel microRNA regulator of prostate cancer epithelial-mesenchymal transition
}

\author{
Nathan Bucay ${ }^{1}$, Divya Bhagirath ${ }^{1}$, Kirandeep Sekhon ${ }^{1}$, Thao Yang ${ }^{1}$, Shinichiro Fukuhara ${ }^{1}$, Shahana Majid ${ }^{1}$, Varahram Shahryari ${ }^{1}$, \\ Z Laura Tabatabai ${ }^{2}$, Kirsten L Greene ${ }^{1}$, Yutaka Hashimoto ${ }^{1}$, Marisa Shiina ${ }^{1}$, Soichiro Yamamura ${ }^{1}$, Yuichiro Tanaka ${ }^{1}$, Guoren Deng ${ }^{1}$, \\ Rajvir Dahiya ${ }^{1}$ and Sharanjot Saini ${ }^{*} 1$
}

The most frequent alteration in the prostate oncogenome is loss of chromosome (chr) 8p21 that has been associated with loss of NKX3.1 homeobox gene. Chr8p21 deletions increase significantly with tumor grade and are associated with poor prognosis in prostate cancer $(\mathrm{PCa})$, suggesting critical involvement of this region in tumor progression. Recent studies suggest that apart from NKX3.1, this region harbors alternative tumor suppressors that are yet undefined. We proposed a novel, paradigm shifting hypothesis that this locus is associated with a miRNA gene cluster-miR-3622a/b- that plays a crucial suppressive role in PCa. Here we demonstrate the crucial role of miR-3622a in prostate cancer epithelial-to-mesenchymal transition (EMT). MicroRNA expression profiling in microdissected human PCa clinical tissues showed that miR-3622a expression is widely downregulated and is significantly correlated with poor survival outcome and tumor progression. To understand the functional significance of miR-3622a, knockdown and overexpression was performed using non-transformed prostate epithelial and PCa cell lines, respectively, followed by functional assays. Our data demonstrate that endogenous miR-3622a expression is vital to maintain the epithelial state of normal and untransformed prostate cells. miR-3622a expression inhibits EMT, progression and metastasis of $\mathrm{PCa}$ in vitro and in vivo. Further, we found that miR-3622a directly targets EMT effectors ZEB1 and SNAI2. In view of these data, we propose that frequent loss of miR-3622a at chr8p21 region leads to induction of EMT states that in turn, promotes PCa progression and metastasis. This study has potentially significant implications in the field of prostate cancer as it identifies an important miRNA component of a frequently lost chromosomal region with critical roles in prostate carcinogenesis which is a highly significant step towards understanding the mechanistic involvement of this locus. Also, our study indicates that miR-3622a is a novel PCa biomarker and potential drug target for developing therapeutic regimens against advanced PCa.

Cell Death and Differentiation (2017) 24, 1263-1274; doi:10.1038/cdd.2017.69; published online 12 May 2017

Epithelial-mesenchymal transition (EMT), the initiating and most critical step in invasion and metastasis, is characterized by decreased expression of epithelial genes (such as E-cadherin) and increased expression of mesenchymal genes (such as vimentin). ${ }^{1-3}$ It is a complex genetic program involving interactions of diverse inducers (for example, transforming growth factor- $\beta$, (TGF- $\beta$ )), multiple signaling pathways and is coordinated by EMT-transcription factors (TFs) (such as SNAIL, ZEB families). In prostate cancer (PCa), EMT has been implicated particularly in the context of metastatic disease. ${ }^{4-7} \mathrm{E}$-cadherin expression is commonly lost or reduced in $\mathrm{PCa}^{4,5}$ and its loss along with gain of $\mathrm{N}$-cadherin has been associated with multiple end points of progression and mortality. ${ }^{8}$ The existence of sarcomatoid prostate carcinomas with a metastatic propensity ${ }^{9}$ supports the existence of EMT like states in PCa. Though a role for EMT in promoting advanced $\mathrm{PCa}$ has been realized, a well delineated mechanistic model has been lacking. Recently, microRNAs have emerged as key regulators of $\mathrm{EMT}^{10}$ and progression and metastasis of human cancers. ${ }^{11-13}$ MicroRNAs (miRNAs) are small RNAs that suppress gene expression post-transcriptionally via sequence-specific interactions with the $3^{\prime}$ - untranslated regions (UTRs) of cognate mRNA targets. ${ }^{14}$ Prominent examples are the miR-200 family and miR-205 that regulate EMT through direct targeting of ZEB1, ZEB2 ${ }^{11,15-21}$ amongst other targets. ${ }^{18}$ We showed that miR-203 regulates $\mathrm{PCa}$ EMT and metastasis by directly targeting ZEB2 and BMI1. 5,22 Signaling pathways implicating miRNAs in PCa EMT are yet to be fully deciphered.

Prostate cancer genome annotation have consistently reported chromosome $8 p$ (chr8p) loss, particularly that of chr8p21 sub region, as a frequent alteration. ${ }^{23-29}$ This region is associated with a high rate of $\mathrm{LOH}^{30,31}$ and harbors prostate-specific NKX3.1 gene. ${ }^{32}$ A significantly higher deletion frequency has been reported in advanced $\mathrm{PCa},{ }^{33}$ suggesting its role in disease progression. Chr8p losses were shown to occur in $55.7 \%$ cases of localized and $90.5 \%$ cases of advanced $\mathrm{PCa}^{34}$ These deletions increase significantly with tumor grade, ${ }^{35}$ are associated with tumor progression and poor prognosis. ${ }^{36}$ Further, genomic studies suggest that copynumber loss of this region does not correlate with NKX3.1 mRNA expression, ${ }^{29}$ suggesting the possibility of alternative tumor suppressors within this region. Despite its importance, the precise genetic elements within this region have not been

\footnotetext{
${ }^{1}$ Department of Urology, Veterans Affairs Medical Center and University of California, San Francisco, CA, USA and ${ }^{2}$ Department of Pathology, Veterans Affairs Medical Center and University of California San Francisco, San Francisco, CA, USA

${ }^{*}$ Corresponding author: S Saini, Department of Urology, Veterans Affairs Medical Center, University of California San Francisco, 4150 Clement Street, San Francisco, CA 94121, USA. Tel: +415-221-4810 (X23548); Fax: +415-750-6639; E-mail: Sharanjot.Saini@ucsf.edu

Received 10.11.16; revised 13.3.17; accepted 04.4.17; Edited by Y Shi; published online 12.5.17
} 
characterized to date, which is a critical drawback. We identified that a miRNA gene cluster - miR - 3622a/b- is located within this region that is widely down regulated in $\mathrm{PCa}$. We recently reported a tumor suppressive role of miR-3622b in PCa via its regulation of epidermal growth factor receptor. ${ }^{37}$ Towards deciphering the regulatory role of this miRNA cluster in $\mathrm{PCa}$, we explored the role of miR-3622a in the present study and discovered its important regulatory role in PCa EMT. miR-3622a is a recently discovered miRNA gene that has not been studied. ${ }^{38,39}$ Here we provide the first direct evidence that miR-3622a-5p (major form of miR-3622a, referred to as miR-3622a) inhibits PCa EMT, progression and metastasis. This is a novel study that connects the long-standing observation of frequent loss of a chromosomal region with a novel miRNA in PCa.

\section{Results}

Frequent genomic loss of miR-3622a at chr8p21 in PCa. We identified that miRNA gene miR-3622a is located in the deleted chr8p21 region (Figure 1a). ${ }^{38,39}$ We queried copy number alterations (CNAs) at this miRNA locus in $\mathrm{PCa}$ in The Cancer Genome Atlas (TCGA) dataset using cBioportal $^{40,41}$ and found that miR-3622a is frequently deleted either homozygously or heterozygously in $\sim 50 \%$ PCa cases (similar to NKX3.1) (Figure 1b). Interestingly, CNAs at the miR-3622a locus correlated with that of NKX3.1 $(r=0.5345, P<0.0001)$. We hypothesized that miR-3622a gene is frequently lost in $\mathrm{PCa}$ and may underlie $\mathrm{PCa}$ aggressiveness.

miR-3622a expression is widely attenuated in PCa. We performed expression profiling in laser capture microdissected (LCM) PCa tissues $(n=138)$ and matched adjacent normal regions by real-time PCR (Figure 2a). Clinicopathological characteristics of the patients are summarized in Supplementary Table S1. miR-3622a expression was down regulated in $\sim 70 \%$ of tissue samples (Wilcoxon signed rank test, $P<0.001)$. We also performed in silico analyses of miR-3622a expression for primary PCa cases in the TCGA

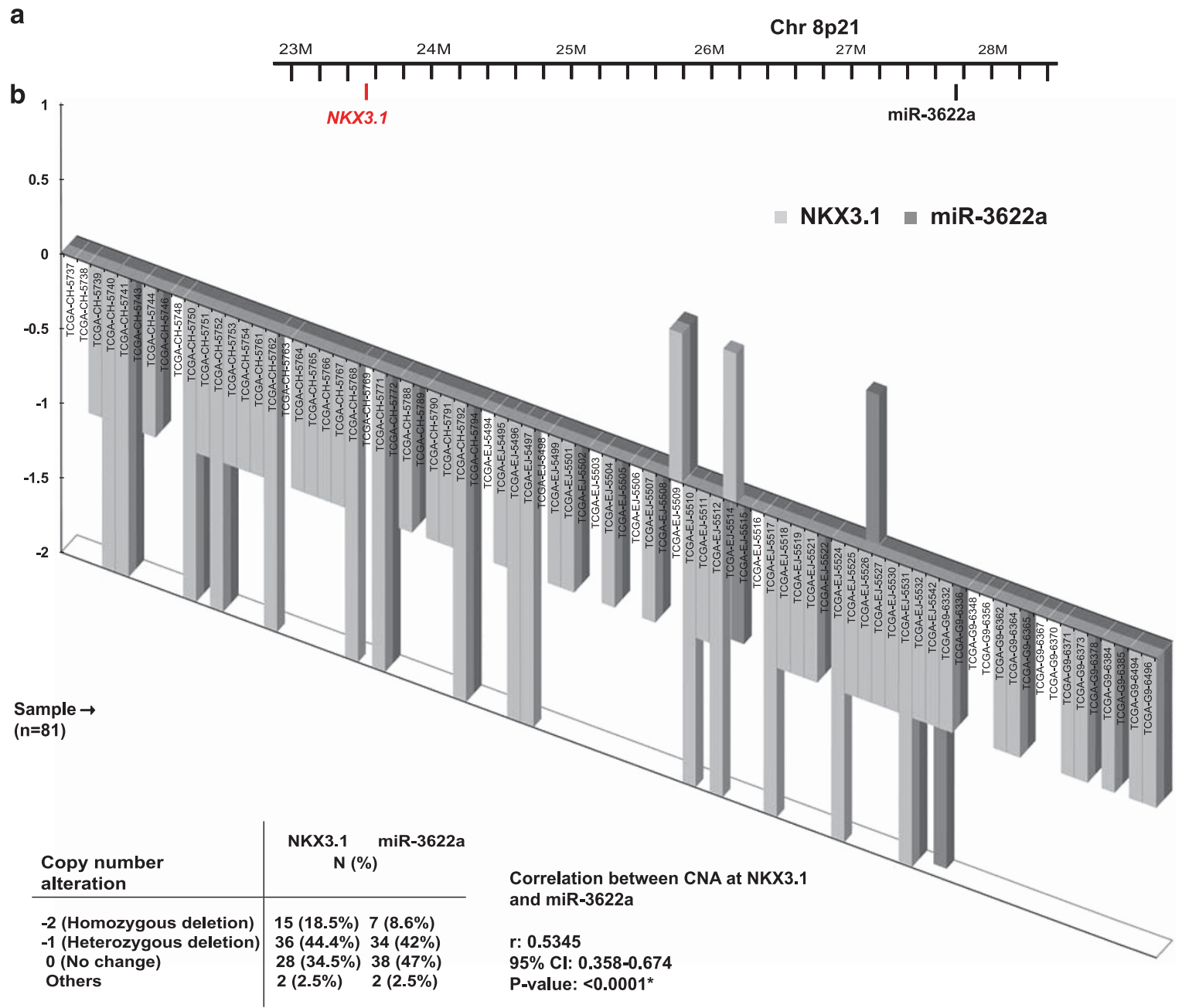

Figure 1 Frequent genomic loss of miR-3622a at chr8p21 in prostate cancer. (a) Schematic representation of chr8p21 region highlighting the genomic location of the miR-3622a locus and NKX3.1. (b) CNAs at miR-3622a and NKX3.1 loci in prostate adenocarcinomas in the TCGA dataset. Table below summarizes the observed CNAs at these loci 
a

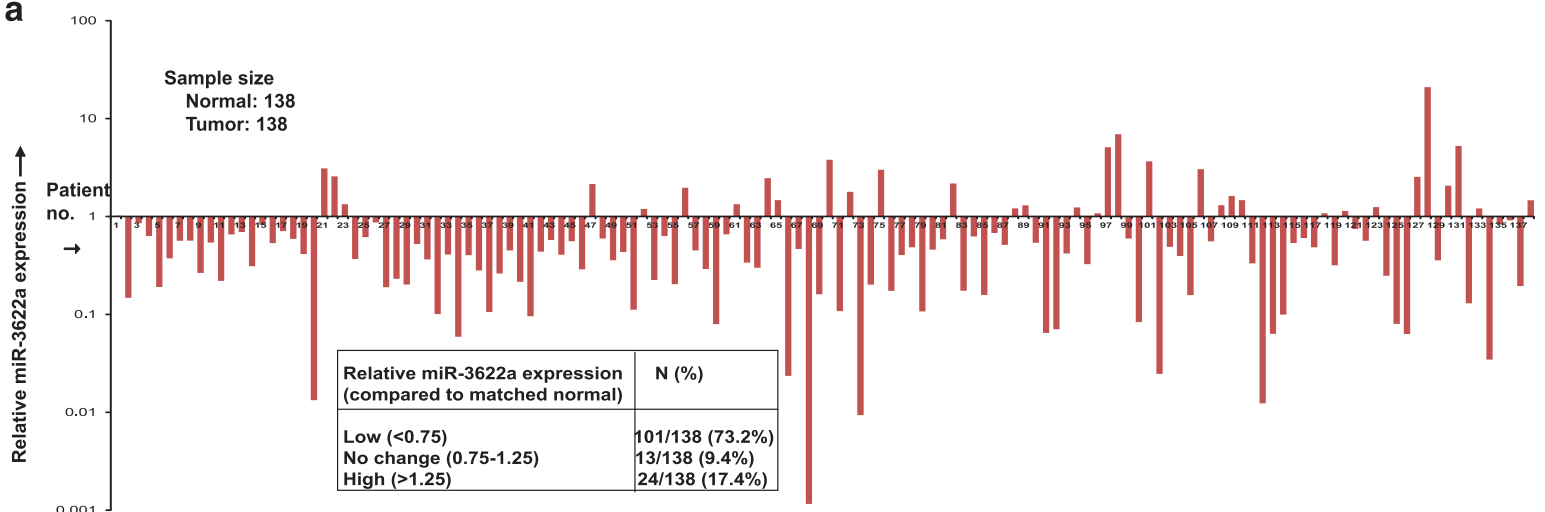

d

" Gleason grade unknown for 4 cases, pathological stage for 16 cases, biochemical recurrence for 14 samples in SFVAMC cohort ..... Clinical data available for 117 samples in TCGA cohort. Biochemical recurrence unknown for 14 samples in TCGA cohort

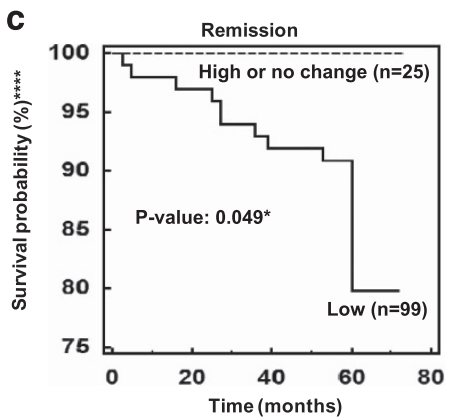

**** Survival data unavailable for 14 samples

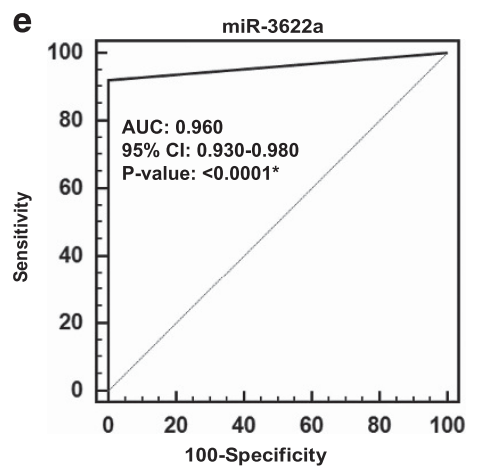

Figure 2 miR-3622a expression is widely attenuated in prostate cancer and low miR-3622a expression is associated with tumor progression and poor survival outcome. (a) Relative miR-3622a expression levels in microdissected PCa tissues $(n=138)$ and patient matched adjacent normal regions as assessed by real-time PCR. The data were normalized to RNU48 control. Table below summarizes the relative miR-3622a expression levels in tumor specimens. (b) Relative miR-3622a expression levels in the TCGA cohort ( $n=184)$. Z-scores were calculated for tumor tissues and plotted along x-axis. (c) Kaplan-Meier survival curves for PCa patients, stratified based on miR-3622a levels. $P$-value based on a log rank test. (d) Correlation of miR-3622a expression with clinicopathological characteristics of PCa patients in SFVAMC and TCGA cohorts. $P$-values were calculated by $\chi^{2}$ test for trend while that for biochemical recurrence represent $\chi^{2}$ test. (e) ROC curve analysis showing the ability of miR-3622a expression to discriminate between malignant and non-malignant tissue samples. (f) Diagnostic utility measures of miR-3622a as a PCa biomarker. $\left({ }^{\star} P<0.05\right)$

dataset ( $n=184$ available at the time of download), (Figure 2b) which showed that miR-3622a expression is attenuated in $\sim 72 \%$ of tumor tissues $(P<0.001)$. These results suggest that miR-3622a expression is widely attenuated in PCa and is inversely associated with the disease.

Low miR-3622a expression is associated with tumor progression and poor survival outcome in PCa. In view of the observed widespread downregulation of miR-3622a, we evaluated the potential clinical significance of miR-3622a expression in PCa. Kaplan-Meier survival analysis for $\mathrm{PCa}$ patients, stratified based on miR-3622a levels (low expression and high/no change groups), showed that overall survival was significantly reduced in patients with low miR-3622a expression (Figure 2c). Correlation of miR-3622a with clinicopathological characteristics in our clinical cohort and TCGA cohort (Figure 2d) suggested that low miR-3622a expression significantly correlated with higher Gleason grade tumors $\left(x^{2}\right.$ test for trend, $\left.P=0.0223^{\star}\right)$. Low miR-3622a expression correlated with higher pathological stage in our clinical cohort though the correlation was statistically insignificant $(P=0.6471)$. However, no correlation 
was observed between miR-3622a expression and biochemical recurrence. Further, ROC (receiver operating characteristic) analyses showed that miR-3622a expression can be a single significant parameter to discriminate between normal and tumor tissues with an area under the ROC curve (AUC) of 0.960 (95\% Cl: 0.930-0.980, $P<0.0001$ ) (Figure 2e). Our analyses showed that miR-3622a exhibits $92 \%$ sensitivity, $100 \%$ specificity, $100 \%$ positive predictive value and $93 \%$ negative predictive value (Figure 2f). Overall, our analyses suggest that miR-3622a has potential as a prognostic and diagnostic PCa marker.

miR-3622a expression is not correlated with NKX3.1 expression in PCa. Further, we examined whether miR-3622a expression is correlated with that of NKX3.1 expression in the TCGA cohort (TCGA had the data for 52 tumors with miRNA-seq and the mRNA expression data at the time of download) (Supplementary Figure S1A). Our analyses suggests that there is no statistically significant correlation between the expression of these two genes, though the CNAs at these two loci are highly correlated (Figure 1b). We also examined NKX3.1 transcript levels in our clinical cohort (Supplementary Figure S1B) and found that the expression of miR-3622a is not correlated with that of NKX3.1 (Supplementary Figure S1B-C). This suggests that the NKX3.1 and miR-3622a loci are regulated independently and may play distinct roles in $\mathrm{PCa}$.

\section{Genomic loss and promoter hypermethylation underlies} low miR-3622a expression in PCa. TCGA cohort showed a genomic loss of one or both miR-3622a loci in $~ 8.6$ and $\sim 42 \%$ of PCa samples, respectively (Figure $1 \mathrm{~b}$ ), while low miR-3622a expression was observed in $\sim 72 \%$ of tumors (Figure 2b). Correlation analyses of CNAs versus miR-3622a expression (Figure 3a) suggested that miR-3622a locus may be subject to additional regulatory controls. Samples with a diploid miR-3622a locus $(C N A=0)$ also showed low expression apart from the cases showing homozygous/ heterozygous loss (CNA $=-2$ or -1$)$. On the basis of this, we hypothesized that epigenetic events co-regulate miR-3622a expression. miR-3622a expression analyses in an immortalized non-transformed prostate epithelial cell line (BPH1) and PCa cell lines (PC3, LNCaP, Du145) (Figure 3b, lower left panel) showed that its expression is significantly reduced in $\mathrm{PCa}$ cells. Examination of the miR-3622a promoter region showed that its upstream region (around -2500 ) is enriched in CpG sites (Figure 3b, upper panel). Methylation-specific PCR (MS-PCR) analyses of this region showed that PCa cell lines exhibit miR-3622a hypermethylation as compared to $\mathrm{BPH} 1$ (Figure 3b, lower right panel), which is consistent with the observed expression, suggesting that this locus is epigenetically downregulated in $\mathrm{PCa}$ cells. To confirm miR-3622a promoter hypermethylation, we treated PC3 cells with DNA demethylating agent, 5-Aza-2'-deoxycytidine (5-Aza) followed by MS-PCR (Figure 3c, right panels) and real-time PCR analyses (Figure 3c, left panel). Our analyses showed that 5-Aza treatment led to decreased promoter methylation concomitant with increased miR-3622a expression. We also performed methylation, CNA and expression analyses on a subset of our clinical cohort (Figure 3d).
MS-PCR (Figure 3d, upper panel) and CNA analyses (Figure 3d, lower left panel) showed that the miR-3622a upstream region is hypermethylated in 6/11 ( 55\%) tumor samples (indicated by arrows) and that $8 / 11$ tissues $(73 \%)$ exhibit decreased relative levels of copy number compared to matched normals. miR-3622a expression levels (RT-PCR) are shown in Figure 3d, lower right panel. Our analyses show that methylation was mostly increased, particularly in tumor samples showing no loss or loss of one copy of miR-3622a gene, suggesting that genetic and epigenetic factors coordinately regulate miR-3622a expression in $\mathrm{PCa}$.

miR-3622a overexpression inhibits EMT and reduces invasiveness of PCa cell lines. To assess the functional significance of $\mathrm{miR}-3622 \mathrm{a}$ in $\mathrm{PCa}$, we overexpressed miR-3622a/ control miR (miR-CON) in PCa cell lines (PC3, LNCaP, Du145) (Figure 4a) by transient transfection of miRNA precursors followed by functional assays. The rationale for selecting these cell lines was to gauge the effects of miR-3622a overexpression using both androgendependent (LNCaP) and androgen-independent (PC3, Du145) cell lines. miR-3622a overexpression significantly decreased the invasiveness (Figure 4b) and migration (Figure $4 \mathrm{c}$ ) of $\mathrm{PCa}$ cell lines, suggesting a critical role for this miRNA in determining the invasive phenotype. miR-3622a overexpression led to marked morphological changes with a decrease in the fraction of elongated, spindle-shaped cells paralleled by an increase in rounded, adherent cells indicating transition from a fibroblast- to epithelial-like phenotype (Figure $4 d$, upper panels). The morphological changes induced by miR-3622a reexpression were assessed by examining the actin cytoskeletal organization by FITC-labelled phalloidin staining (Figure 4d, lower panels). Our data suggest that miR-3622a induces substantial cytoskeletal reorganization with most miR-3622a expressing cells presenting a predominantly cortical actin distribution with a concomitant loss of filopodia as compared to control. Upon miR-3622a expression, cells displaying epithelial characteristics increased from 11 to $93 \%$ in PC3 cells and 7 to $86 \%$ in LNCaP cells (Figure 4e). Immunoblotting showed that miR-3622a overexpression resulted in increased E-cadherin expression with a concomitant decrease in vimentin (Figure 4f), suggesting that miR-3622a promotes the epithelial phenotype and inhibits EMT. Increased expression of E-cadherin was confirmed by immunofluorescent staining of PC3 and LNCaP cells upon miR-3622a transfection as compared to control cells (Figure $4 \mathrm{~g}$ ). We also found a positive correlation between miR-3622a and E-cadherin expression in a subset of our clinical cohort $(n=18)$ by E-cadherin immunohistochemical staining (Figure $4 \mathrm{~h})$.

miR-3622a knockdown induces EMT and increases invasiveness of normal and non-transformed prostate epithelial cells. To further understand the role of miR$-3622 a$, we asked if its knockdown in untransformed primary prostate epithelial cells (PPEC) or semi-transformed prostate epithelial cells (BPH1), in addition to their overexpression in $\mathrm{PCa}$ cell lines, affects various cellular functions. Therefore, we knocked down miR-3622a endogenous expression in 


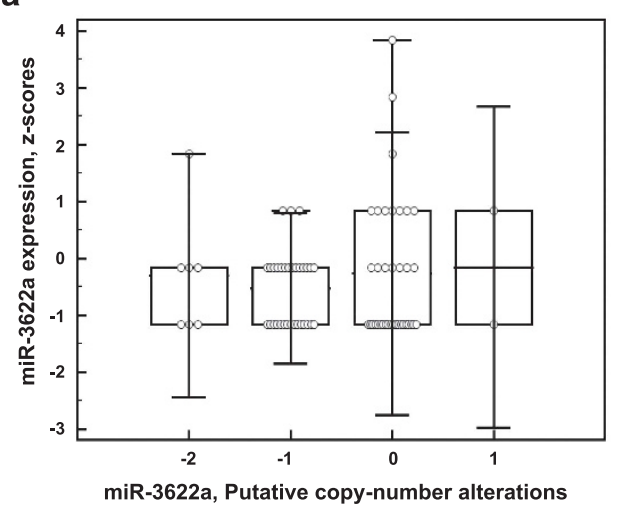

b
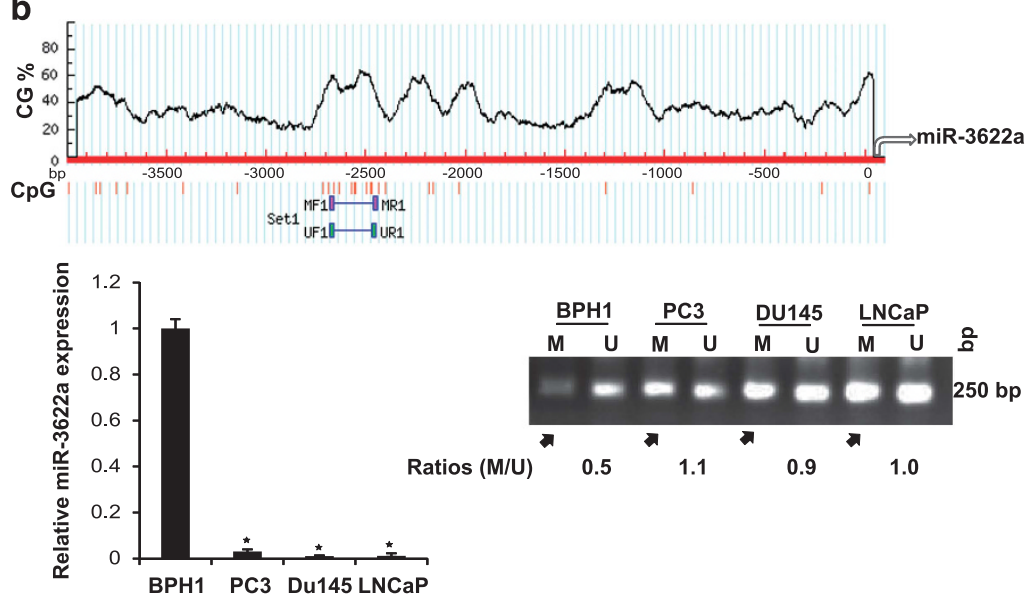

C

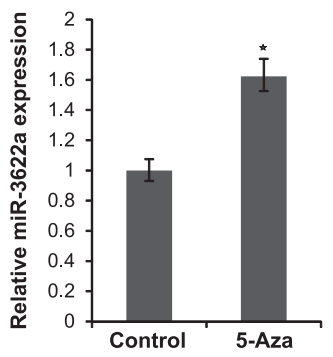

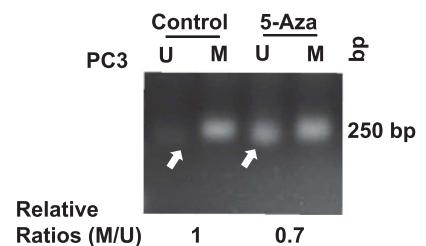

d

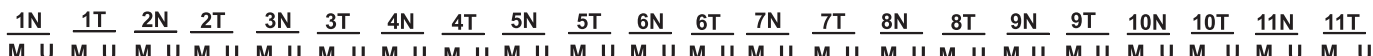

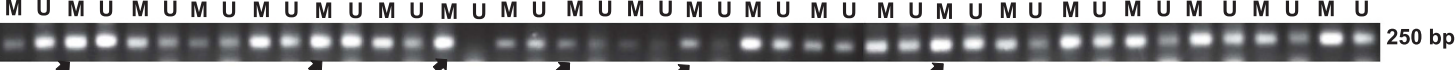

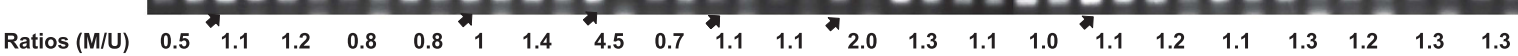
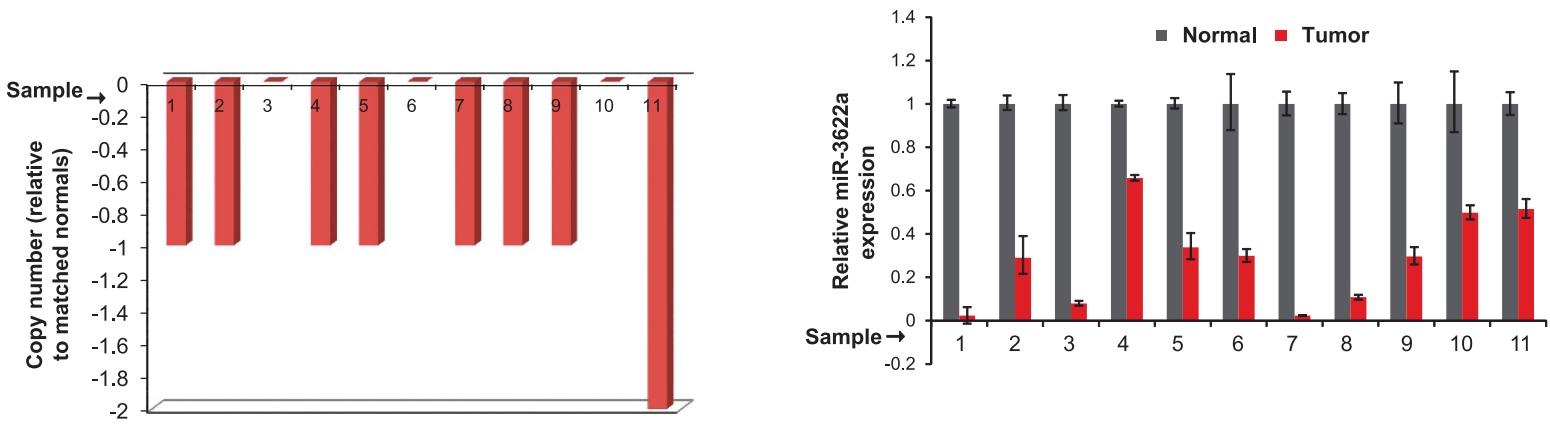

Figure 3 Genomic loss and promoter hypermethylation of miR-3622a locus underlies low miR-3622a expression in prostate cancer. (a) Box plot of miR-3622a expression versus CNAs at the locus in the TCGA cohort of PCa tissues. (b) Upper panel: Schematic representation showing the CpG-rich region upstream of miR-3622a gene. Percentage of $\mathrm{C}+\mathrm{G}$ nucleotides (\%CG) is shown and the density of $\mathrm{CpG}$ nucleotides is shown for a $\sim \mathrm{kb}$ region upstream of miR-3622a. The upstream region (around -2500 ) is enriched in $\mathrm{CpG}$ sites. The position of primers used for MS-PCR analyses of miR-3622a upstream region are depicted schematically below. Lower panel (left): Relative miR-3622a expression levels in prostate cell lines. The data were normalized to RNU48 control and are represented as mean \pm S.E.M. Lower panel (right): MS-PCR analysis of miR-3622a $5^{\prime}$ upstream region in prostate cell lines showing methylated (M) or unmethylated (U) DNA products. Arrows indicate methylation at the miR-3622a locus. Ratios below represent the quantification of intensities of methylated relative to unmethylated products. (c) Real-time PCR expression analyses (left panel) and MS-PCR analyses (right panels) of miR-3622a locus in PC3 cells treated with vehicle control (DMSO) or $1 \mu \mathrm{M}$ of 5-Aza-2'-deoxycytidine (5-Aza) for 4 days. Ratios below represent the quantification of intensities of methylated relative to unmethylated products. (d) MS-PCR analyses (upper panel), CNA analyses (lower left) and real-time PCR expression analyses (lower right) of miR-3622a locus in PCa clinical tissues. Ratios below represent the quantification of intensities of methylated relative to unmethylated products for tumor samples with matched adjacent normal. Hypermethylation at the miR-3622a locus in tumor samples is indicated by arrows

PPEC and $\mathrm{BPH}_{1}{ }^{42}$ (Figure 5a), which led to marked morphological changes, suggesting transition from an epithelial- to mesenchymal-like phenotype (Figure 5b). FITC-labelled phalloidin staining (Figure 5b, lower panels) showed that miR-3622a knockdown induces alterations consistent with EMT induction such as gain of filopodia and loss of cortical actin distribution. In BPH1 cells, stable miR-3622a knockdown primarily led to loss of cortical actin distribution. Quantification of cells based on epithelial and mesenchymal features showed that upon miR-3622a knockdown, the epithelial fractions decreased significantly (from 88 to $18 \%$ in PPEC; 90 to $10 \%$ in $\mathrm{BPH} 1$ ) with a concomitant 

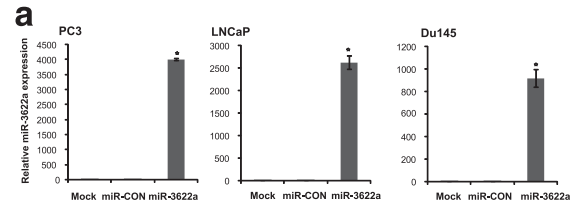

b
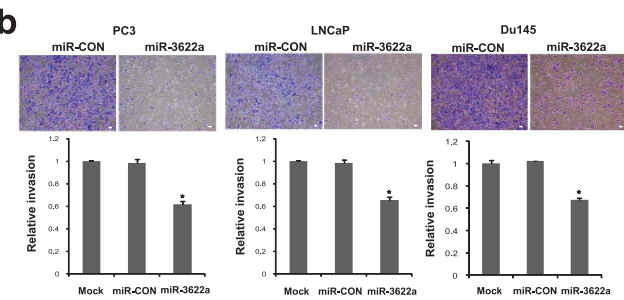

C
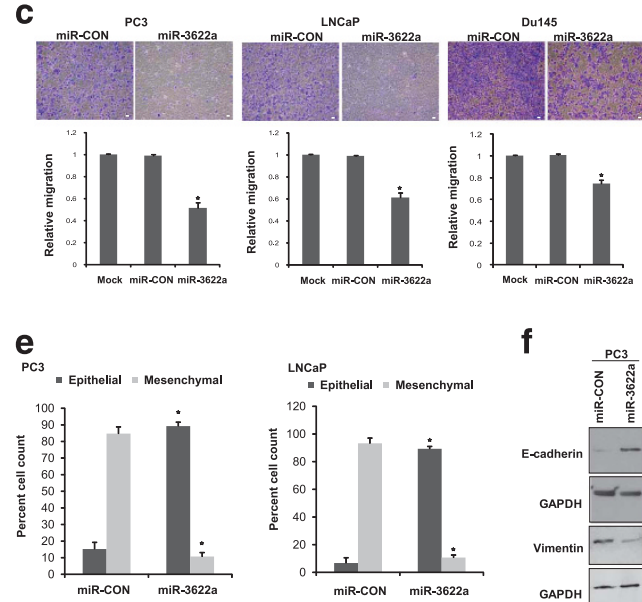

d
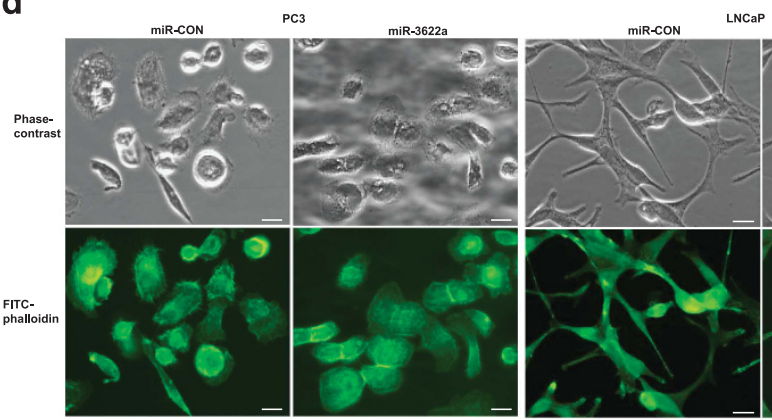

9
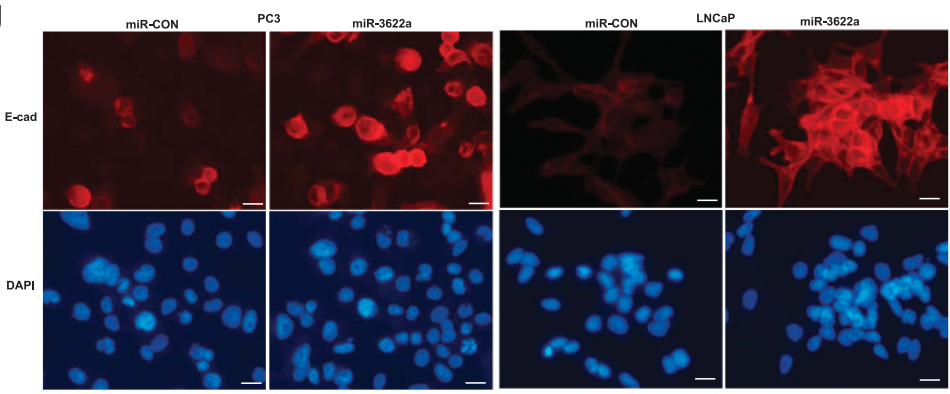

$\mathbf{h}$

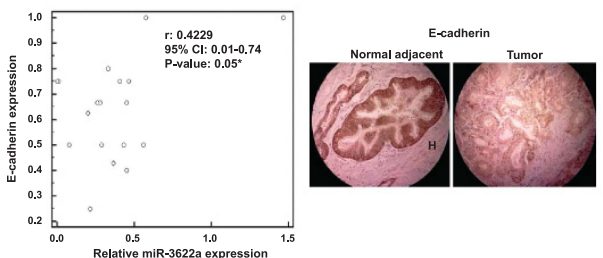

Figure 4 miR-3622a overexpression inhibits EMT and reduces invasiveness of PCa cell lines. Control miR (miR-CON)/miR-3622a was overexpressed in PCa cell lines (PC3, LNCaP, Du145) followed by functional assays $\left({ }^{*} P<0.05\right)$. (a) Relative miR-3622a expression in PC3/LNCaP/Du145 cells transfected with either control miR $/ \mathrm{miR}-3622 \mathrm{a} / \mathrm{mock}$ transfected cells as assessed by real-time PCR. The data were normalized to RNU48 control and are represented as mean \pm S.E.M. (b) Transwell invasion assay in miR-CON/ miR-3622a transfected PC3 (left panels), LNCaP (middle panels), Du145 (right panels) cell lines. Scale bar: $100 \mu \mathrm{m}$. (c) Transwell migration assay in miR-CON/ miR-3622a transfected PC3 (left panels), LNCaP (middle panels), Du145 (right panels) cell lines. Scale bar: $100 \mu \mathrm{m}$. (d) Morphological alterations in PC3 (left panels) and LNCaP cells (right panels) upon miR-CON/ miR-3622a transfections as assessed by phase-contrast microscopy (upper panels). Assessment of actin cytoskeleton in miR-CON/ miR-3622atransfected PC3 and LNCaP cells by FITC-labelled phalloidin staining (lower panels). Magnification 40x. Scale bar $50 \mu$ m. (e) Quantification of cells with epithelial and mesenchymal characteristics is represented by bar graphs (lower panels) as average percent cell count \pm S.D. (f) Immunoblotting of miR-CON/ miR-3622a transfected PC-3 (left panels) and LNCaP (right panels) cells. GAPDH was used as a loading control. (g) Immunofluorescence staining for E-cadherin (red) in miR-CON/miR-3622a transfected PC3 and LNCaP cells. Cells were counterstained with DAPI nuclear stain (blue). Magnification $40 x$. Scale bar: $50 \mu \mathrm{m}$. (h) Left panel: Correlation between E- cadherin expression (as assessed by IHC staining) and miR-3622a expression (as assessed by RT-PCR) in PCa clinical tissues ( $n=18$ ). Right panel: Representative IHC staining of E-cadherin in tumor and matched normal tissue

increase in mesenchymal fractions $(12-82 \%$ in PPEC; $10-90 \%$ in $\mathrm{BPH} 1$ ) as compared to corresponding controls (Figure 5b). Consistent with these alterations, miR-3622a knockdown increased the invasiveness and motility (Figures $5 \mathrm{c}$ and $\mathrm{d}$ ) of these cells. Immunoblotting showed that miR-3622a knockdown led to decreased expression of E-cadherin and Claudin 1 (Figure 5e). Strikingly, PPEC showed a substantial increase in vimentin upon miR-3622a knockdown, demonstrating that the cells had gained mesenchymal characteristics. Overall, our data demonstrate that endogenous miR-3622a expression is vital to maintain the epithelial state of normal/ untransformed prostate cells.

miR-3622a inhibits EMT by direct targeting of EMT effectors in PCa. To identify putative miR-3622a targets, we evaluated the gene expression profiles of PC3 cells transfected with miR-CON/ miR-3622a by microarray analysis. Pathway analysis showed that miR-3622a affects genes related to EMT (Supplementary Figure S2). In silico and microarray analyses (Supplementary Figure S2) identified that key EMT regulators including $\beta$-catenin (CTNNB1), members of the SNAIL (SNAI2, SNAI3) and ZEB families (ZEB1/ZEB2) as putative miR-3622a targets (Supplementary Table S2). To confirm the microarray data, we profiled the expression of putative target genes following miR-3622a modulation by RT-PCR (Figure 6a,Supplementary Figure S3A). miR-3622a knockdown in PPEC/BPH1 cells led to overexpression of ZEB1, SNAI2 and CTNNB1 mRNA, while miR-3622a overexpression in PC3, LNCaP and Du145 cells inhibited their expression (Figure $6 \mathrm{a}$ and Supplementary Figure S3A). Immunoblotting for miR-3622a inhibited $\mathrm{BPH} 1$ cells and miR-CON/miR-3622a overexpressing PC3/Du145/LNCaP 
a

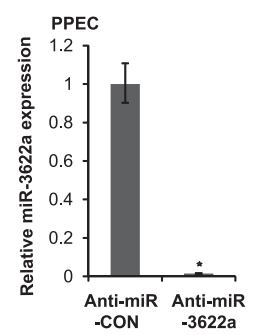

C
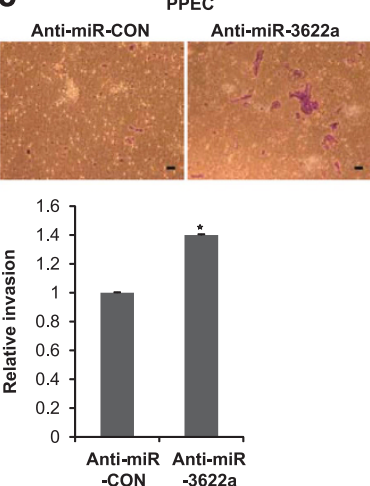

d
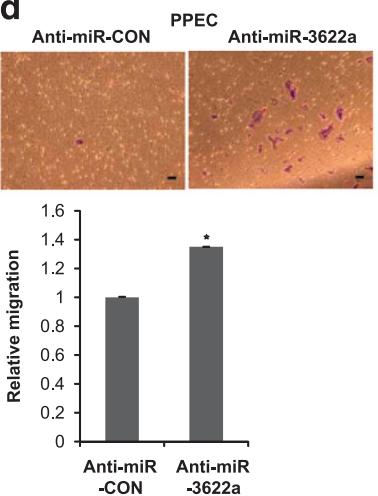
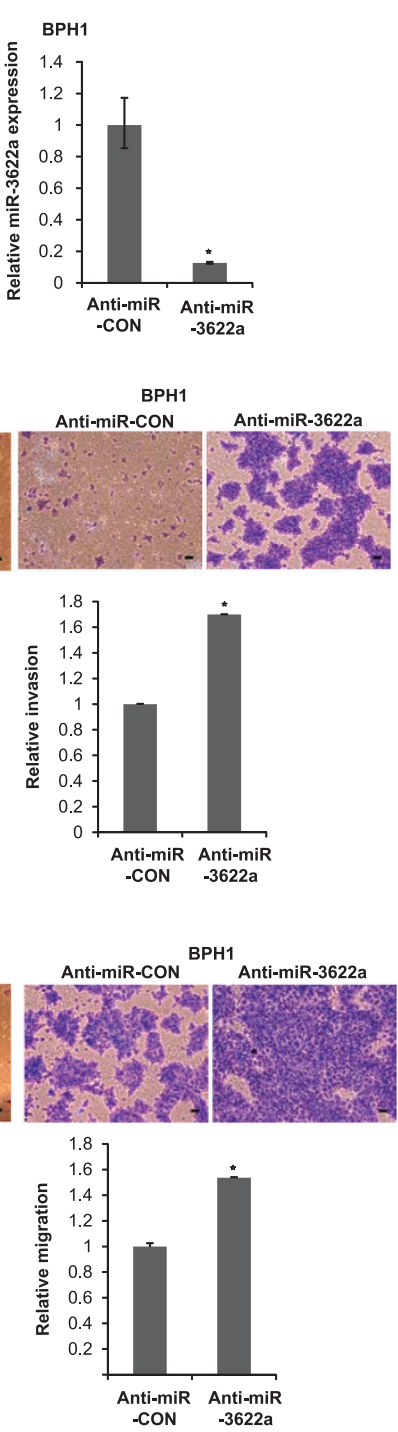

b
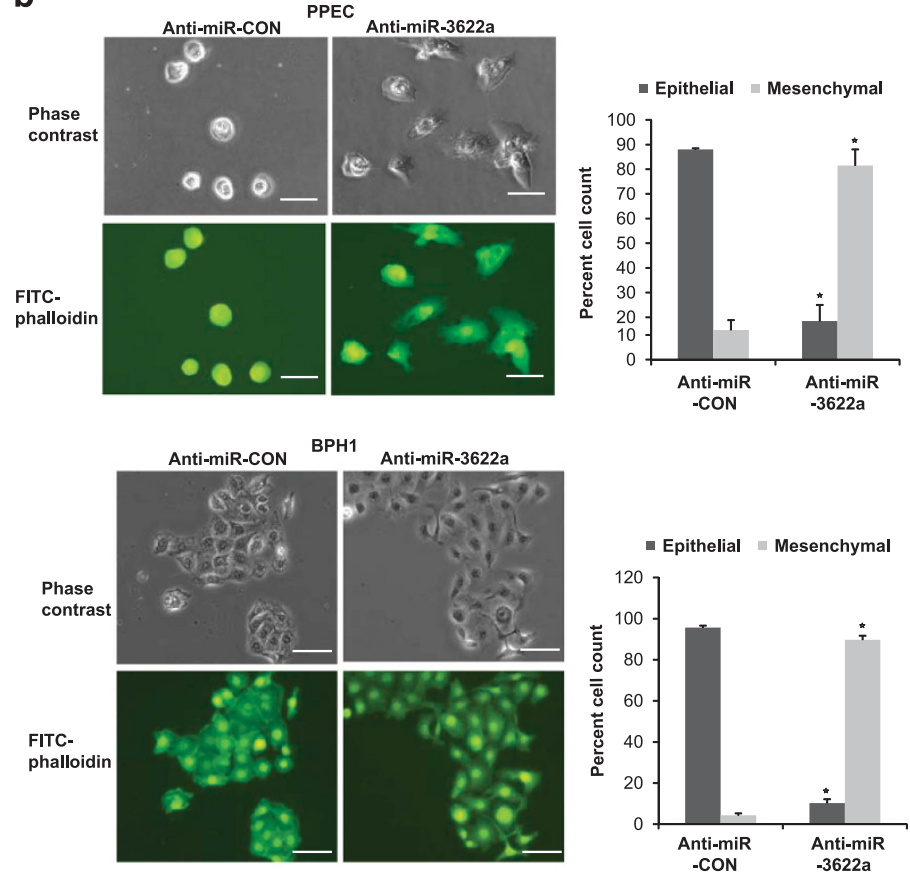

e
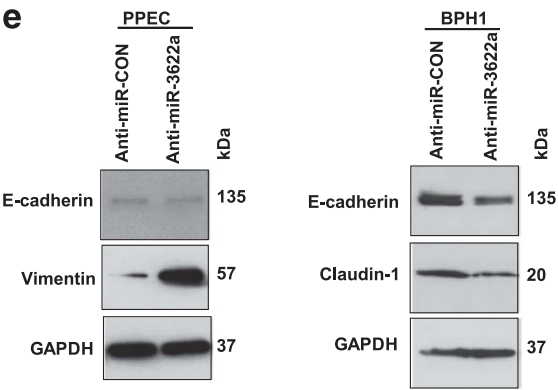

Figure 5 miR-3622a knockdown induces EMT and increases invasiveness of normal and non-transformed prostate epithelial cells. Inhibition of miR-3622a/miR-CON was performed in PPEC and BPH1 cell line followed by functional assays $\left.{ }^{*} P<0.05\right)$. (a) Assessment of miR-3622a expression levels upon knockdown by RT-PCR. The data were normalized to RNU48 control and are represented as mean \pm S.E.M. (b) Morphological alterations upon anti-miR-CON/ anti-miR-3622a treatments as assessed by phasecontrast microscopy (upper panels) and FITC-labelled phalloidin staining (lower panels) in PPEC and BPH1 cells. Magnification $40 \times$. Scale bar: $50 \mu \mathrm{m}$. Quantification of cells with epithelial and mesenchymal characteristics is represented by bar graphs below as average percent cell count \pm S.D. (c) Transwell invasion assay and (d) Transwell migration assay in anti-miR-CON/ anti-miR-3622a-treated PPEC and BPH1 cells. Cells were allowed to migrate/invade for $24 \mathrm{~h}$ at $37^{\circ} \mathrm{C}$. Magnification $10 \times$. Scale bar: $200 \mu \mathrm{m}$. (e) Immunoblots for indicated proteins in PPEC and BPH1 cells treated with anti-miR-CON/ anti-miR-3622a. GAPDH was used a loading control

cells (Figure 6b, Supplementary Figure S3B) confirmed the reciprocal regulation between miR-3622a expression and ZEB1, SNAI2, CTNNB1 protein levels. However, ZEB1 protein could not be detected by immunoblotting in $\mathrm{LNCaP}$ cells due to its low basal levels. The $3^{\prime}$-UTR regions of ZEB1, CTNNB1 possess one potential miR-3622a binding site, while that of SNAI2 has three (Figure $6 \mathrm{c}$ ). To validate these genes as direct targets, we cloned their $3^{\prime}$-UTR regions into luciferase reporter vector and performed reporter assays (Figure 6d) in anti-miRCON/anti-miR-3622a transfected BPH1 cells (Figure 6d, left panel) and miR-CON/miR-3622a transfected PC3/LNCaP cells (Figure $6 \mathrm{~d}$, middle and right panels). Reporter assays with ZEB1/SNAI2/CTNNB1 3'-UTR constructs in miR-3622a inhibited BPH1 cells led to induction of these target genes, while miR-CON/miR-3622a cotransfection in PC3/LNCaP cells repressed their luciferase reporter activity as compared to corresponding control $3^{\prime}$-UTR constructs. To verify that these effects on target genes are because of direct miR-3622a interaction with the corresponding binding sites, we mutated the putative binding site/sites in ZEB1/SNAI/CTNNNB1 $3^{\prime}$-UTRs, which significantly prevented upregulation of the reporters upon miR-3622a inhibition in BPH1 cells and their downregulation upon miR-3622a overexpression in PC3 and LNCaP cells (Figure 6d). To further support the regulatory interplay between miR-3622a and its target genes, we induced the expression of these genes by TGF- $\beta$ treatment and 
a

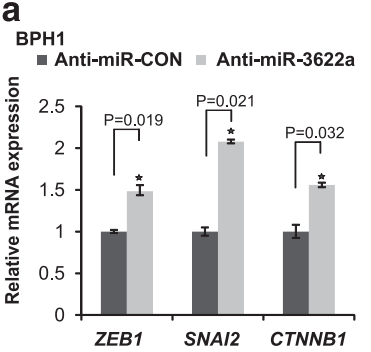

PC3

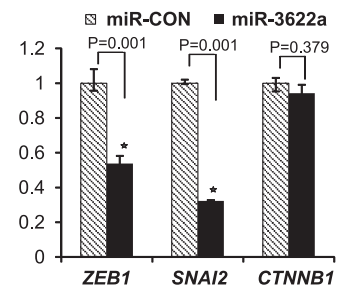

LNCaP

$$
\text { Q miR-CON }=\mathrm{miR}-3622 \mathrm{a}
$$

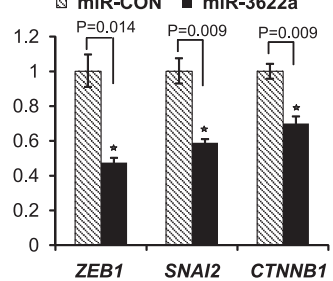

c

$$
\begin{aligned}
& \text { 3' GaguggaCuCGagGGCACGGAC 5' hsa-miR-3622a } \\
& :|||||| \mid \\
& \text { 191: 5'....ACAAAAUAAAUCCGGGUGUGCCUG.... 3' ZEB1 3'UTR } \\
& \text { ACAAAAUAAAUCCGG GAGAACAA mutant ZEB1 } 3 \text { 'UTR } \\
& \text { 3' GAGUGGACUCGAGGGCACGGAC 5' hsa-miR-3622a } \\
& \text { | }|||||| \\
& \text { 382: 5'...GGCUGGUAUCUCAGAAAGUGCCUG....3' CTNNB1 3'UTR } \\
& \text { GGCUGGUAUCUCAGAAAACAAAGA mutant CTNNB1 3'UTR }
\end{aligned}
$$
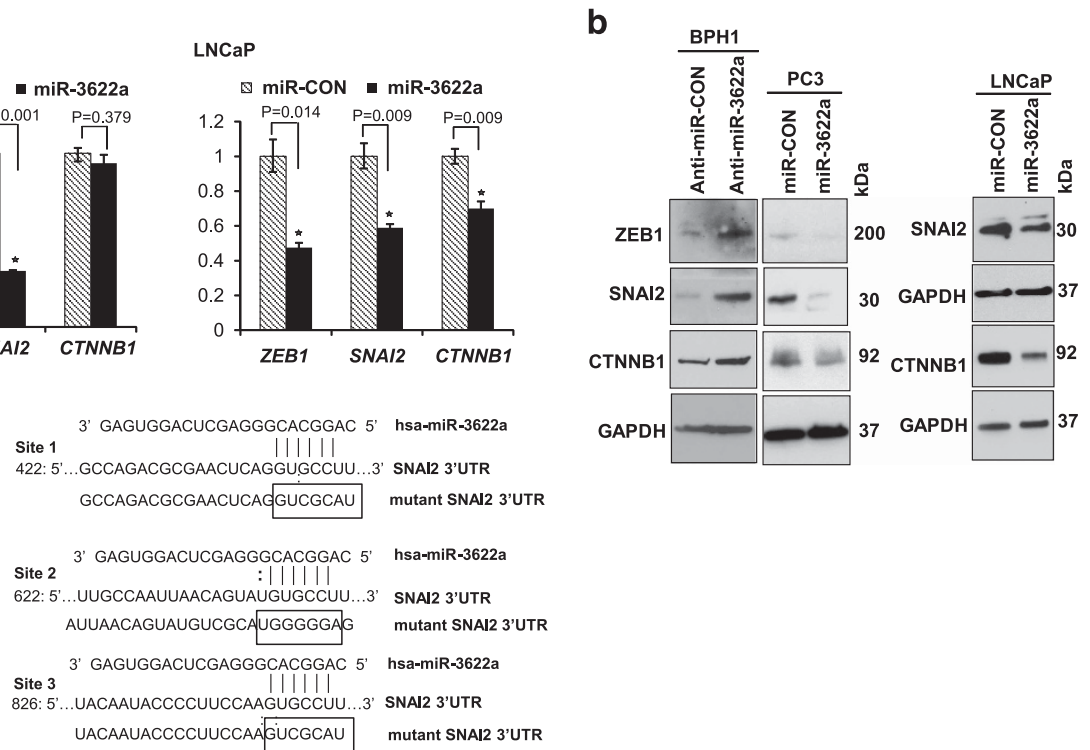

d
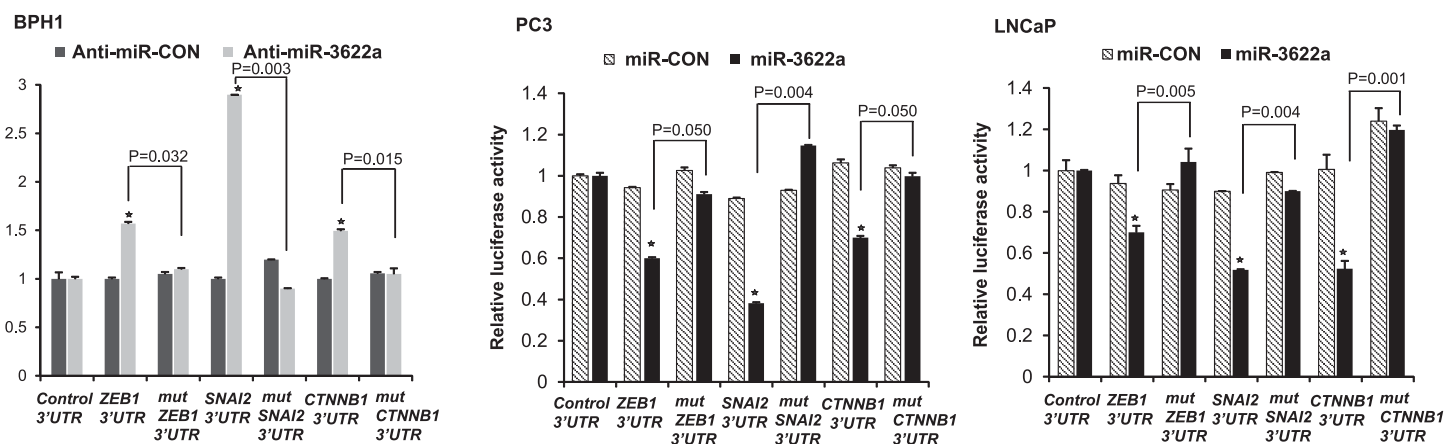

Figure 6 miR-3622a inhibits EMT by direct targeting of EMT effectors in PCa. (a) Relative ZEB1, SNAI2 and CTNNB1 mRNA expression upon indicated treatments in PPEC, BPH1 cells and PCa cell lines (PC3, LNCaP) as assessed by real-time PCR. The data were normalized to GAPDH control. (b)Immunoblots for endogenous ZEB1, SNAI2, CTNNB1 protein in anti-miR-CON/ anti-miR-3622a-transduced BPH1 cells (left panels) and miR-CON/miR-3622a transfected PC3 cells (middle panels). Immunoblots for LNCaP cells are represented in right panels. GAPDH was used a loading control.(c) Schematic representation of ZEB1, CTNNB1, SNAI2 3'-UTRs showing putative miR-3622a target site/sites. For luciferase reporter assays, the potential miR-3622a binding sites were mutated to the sequences shown below. (d) Luciferase reporter assays with the indicated wild type and mutated 3'-UTR constructs or control luciferase construct co-transfected with anti-miR-CON/anti-miR-3622a-transfected BPH1 cells (left panel) and miR-CON/ miR-3622a-transfected PC3 and LNCaP cells (middle and right panels, respectively). Firefly luciferase values were normalized to Renilla luciferase activity and plotted as relative luciferase activity

examined if miR-3622a expression is altered upon TGF- $\beta$ treatment (Supplementary Figure $\mathrm{S} 3 \mathrm{C}$ ). $\mathrm{BPH} 1$ cells were treated with TGF- $\beta$, leading to EMT induction (decreased $C D H 1$ expression and induction of $C D H 2$ and VIM). ZEB1, SNAI2, CTNNB1 expression were induced upon TGF- $\beta$ treatment concomitant with a significant reduction in miR-3622a expression (Supplementary Figure S3C).

ZEB1 and SNAI2 are functionally relevant miR-3622a targets in PCa. We performed phenocopy experiments in PC3 cells by inhibiting ZEB1, SNAI2, CTNNB1 expression using siRNA to see if their knockdown functionally mimics the effects of miR-3622a overexpression (Figure 7a, Supplementary Figure S4A). Knockdown of these genes, particularly ZEB1 and SNAI2, led to morphological alterations (Supplementary Figure S4A), decreased invasiveness and migration (Figures $7 a$ and b) as was observed upon miR-3622a overexpression. To determine if ZEB1 and SNAI2 are functionally relevant miR-3622a targets, we asked if ZEB1 and SNAI2 are the key mediators of EMT induced by miR-3622a inhibition in BPH1 cells (Figures 7c-e). We performed siRNA-mediated knockdown of ZEB1 and SNAI2 in miR-3622a-inhibited BPHI cells (Supplementary Figure S4B). This led to alterations consistent with EMT inhibition, including decreased invasiveness and motility (Figures 7c and d). Consistent with these changes, E-cadherin levels were upregulated/ restored in ZEB1/SNAI2inhibited cells compared to control (Figure 7e). To further validate these genes as direct miR-3622a targets, we also measured their expression levels in a subset of our clinical cohort and found an inverse correlation between ZEB1/ SNAI2 expression and miR-3622a (Figure 7f).

miR-3622a overexpression reduces tumor progression and metastasis in vivo. To examine the in vivo role of miR-3622a in tumor progression and metastasis, 


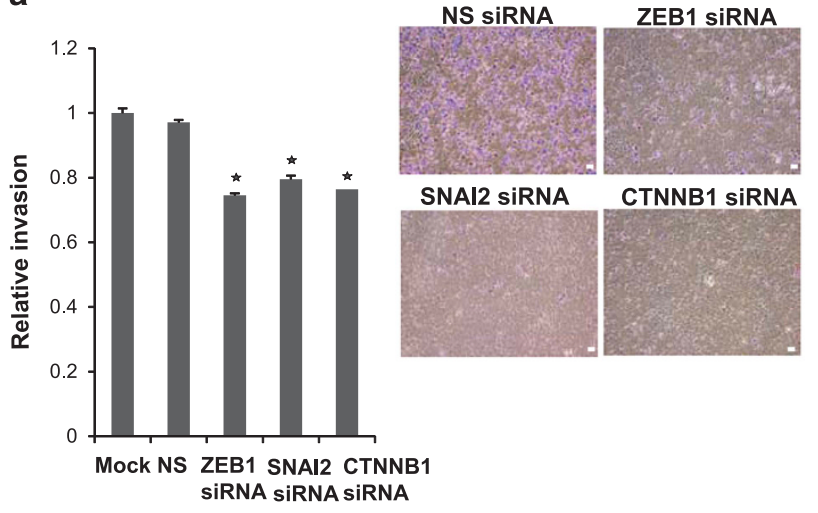

C

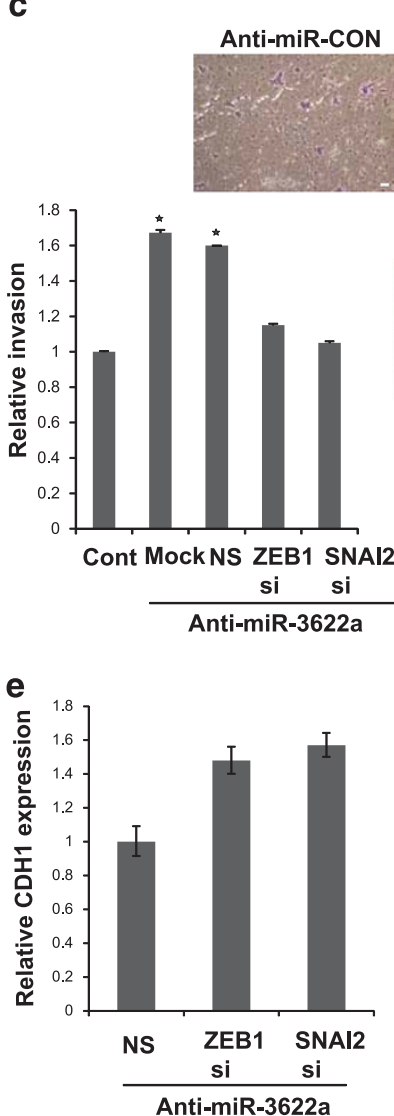

b

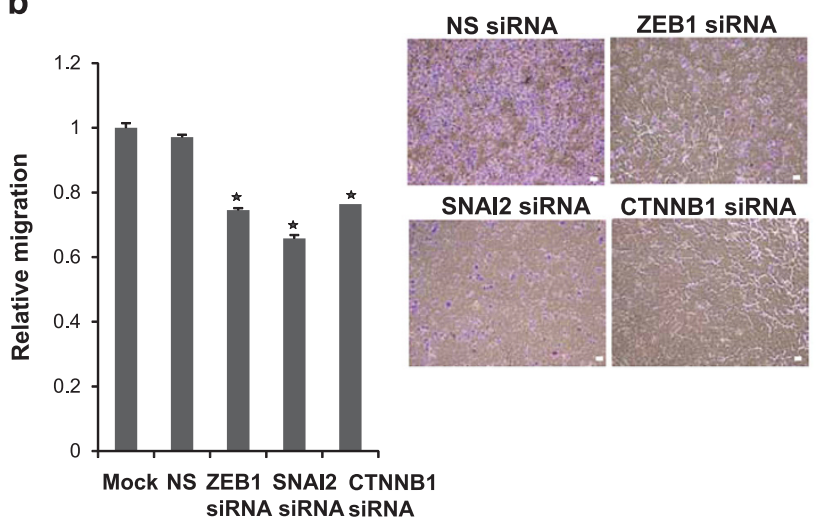

d

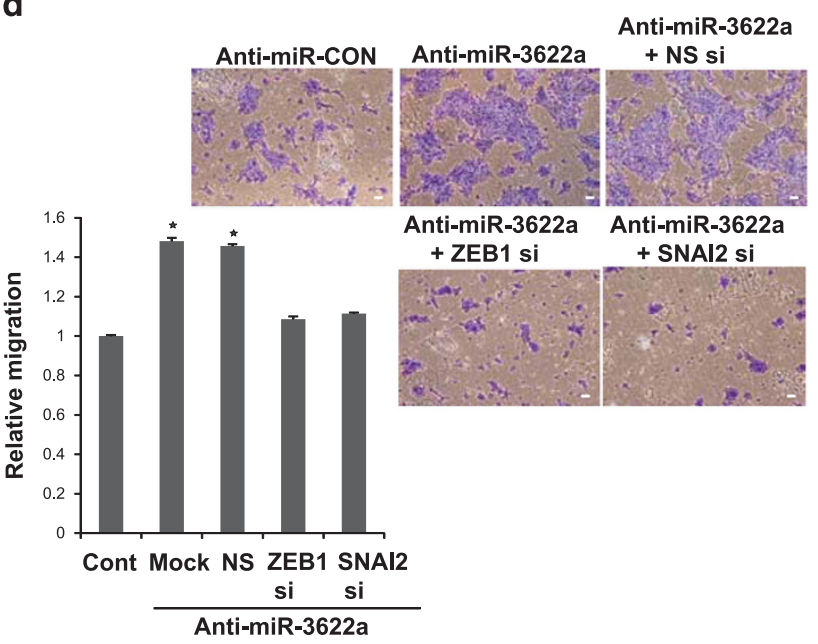

- miR-3622a $\mid$ SLUG

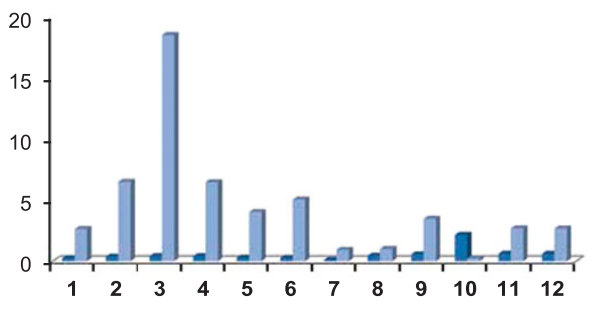

Figure 7 ZEB1 and SNAI2 are functionally relevant targets of miR-3622a in prostate cancer. PC3 cells were transfected with siRNA specific to ZEB1/SNAI2/CTNNB1 or a nonspecific (NS) control siRNA for $72 \mathrm{~h}$ followed by functional assays (a,b). (a) Transwell invasion assay and (b) migration assay in NS/ZEB1/SNAI2/CTNNB1 siRNA-transfected PC3 cells. Scale bar: $100 \mu \mathrm{m}$. To determine whether ZEB1 and SNAI2 are the key mediators of EMT induced by miR-3622a inhibition in the non-transformed BPH1 cell line, we performed siRNA-mediated inhibition of ZEB1 and SNAI2 in miR-3622a-inhibited BPHI cells (Anti-miR-3622a BPHI.) (Supplementary Figure S4B) followed by functional assays (c-e). (c) Transwell invasion assay and (d) migration assay in NS/ZEB1/SNAI2 siRNA-transfected BPH1 cells. Scale bar: $200 \mu \mathrm{m}$. (e) Relative mRNA expression of CDH1 in NS/ ZEB1/SNAI2 siRNA-transfected BPH1 cells as assessed by real time PCR. The data were normalized to GAPDH control. (f) Relative expression levels of ZEB1 and SNAI2 (each normalized to GAPDH) and miR-3622a (normalized to RNU48) as assessed by real-time PCR in prostate cancer clinical tissues

bioluminescent PC-3M-luc cells stably transfected with control miR/miR-3622a (Supplementary Figure S5) were generated and orthotopic PCa (Figures $8 \mathrm{a}$ and b) and experimental metastasis mouse models (Figures $8 \mathrm{c}$ and $\mathrm{d}$ ) were employed. The rationale for selection of mouse models were: (i) Orthotopic $\mathrm{PCa}$ mouse model was employed to examine tumor progression as orthotopic implantation has been considered a more accurate pre-clinical model of the disease, owing to a more accurate representation of the tumor microenvironment. PC-3M-luc cells (control miR/miR-3622a) were implanted orthotopically in the dorsal prostate lobe of nude mice followed by periodic monitoring of tumor 


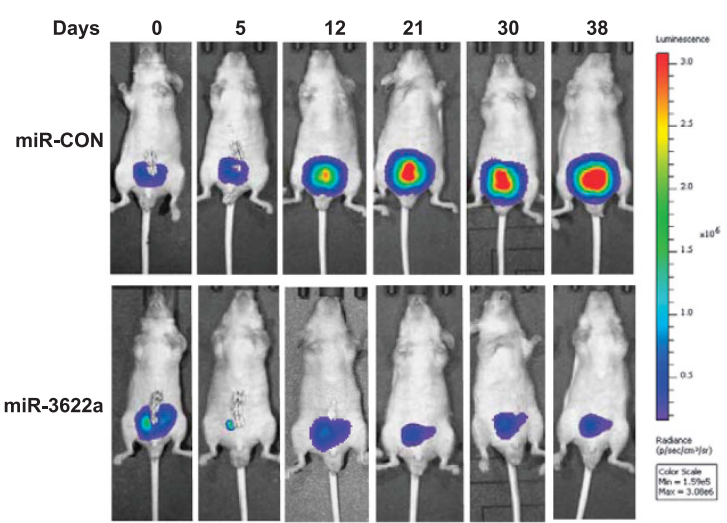

C

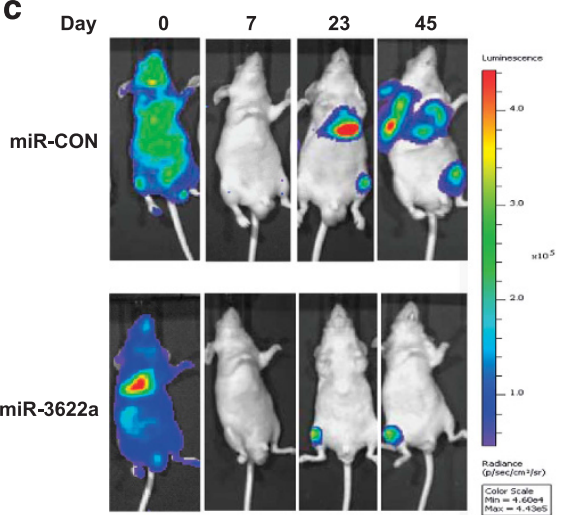

b

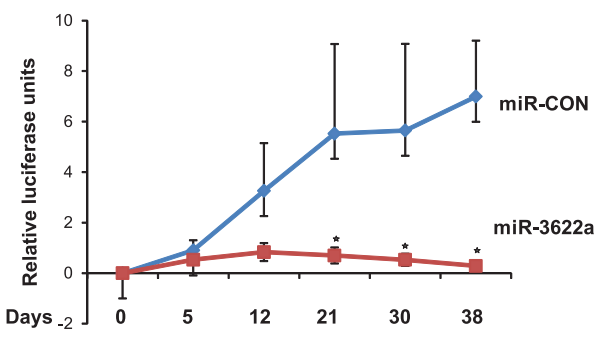

e

Genomic loss, DNA methylation

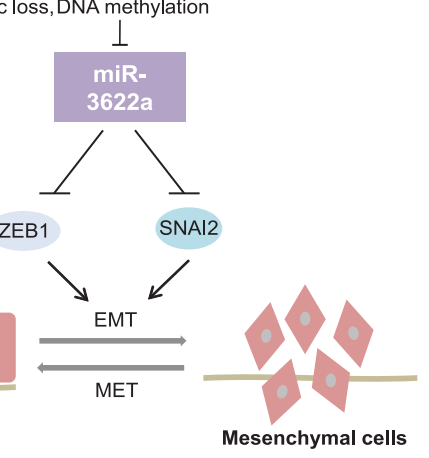

Figure 8 miR-3622a overexpression inhibits prostate cancer progression and metastasis in vivo. (a) Bioluminescent PC-3M-luc cells stably transfected with miR-CON/ miR-3622a were implanted orthotopically in the dorsal prostate lobe of nu/nu mice (day 0) followed by periodic monitoring of tumor development at the indicated time points by in vivo bioluminescent imaging. Representative bioluminescence images from miR-CON (upper panels) and miR-3622a (lower panels) groups at different time-points. The scale bar on the right represents the relative intensity of bioluminescence. (b)Relative growth curves of orthotopic tumors from miR-CON (blue) and miR-3622a (red) groups, with photon counts normalized to values on day 0 . The data represent the mean of each group $\pm S . D\left({ }^{\star} P<0.05\right)$. (c) Bioluminescent PC-3M-luc cells expressing miR-CON/ miR-3622a were injected in the left ventricles of nu/nu mice (day 0 ) followed by periodic monitoring of metastasis at the indicated time points by in vivo bioluminescent imaging. Representative bioluminescence images from miR-CON (upper panels) and miR-3622a (lower panels) groups at different time-points. The scale bar on the right represents the relative intensity of bioluminescence. (d) Quantitation of bioluminescence emitted from whole body of mice on day 45. The data represent the mean of each group \pm S.D $\left({ }^{*} P<0.05\right)$. (e) Schematic representation summarizing the role of miR-3622a-mediated regulatory interactions on EMT outcomes in prostate cancer

development using bioluminescence imaging (Figures 8a and b). Our results suggest that miR-3622a re-expression led to decreased orthotopic tumor growth, confirming the tumor suppressive role of miR-3622a. We also examined the possibility of spontaneous metastases employing this model. However, during the period of the study, significant metastases were not observed. Hence, we employed an experimental metastatic mouse model (below).

(ii) Considering the important role of EMT in the metastatic process and in view of our data, implicating an important role of miR-3622a in EMT, we examined if miR-3622a overexpression affects $\mathrm{PCa}$ metastasis using an experimental metastasis mouse model (Figures $8 \mathrm{c}$ and $\mathrm{d}$ ). This model recapitulates the $\mathrm{PCa}$ metastatic process in vivo and is an excellent model to study PCa bone metastasis. Intracardiac injections of PC-3M-luc cells (control miR/miR-3622a) were performed in nude mice followed by periodic monitoring of the tumor burden in vivo by bioluminescent imaging (Figure 8c). Our data suggest that miR-3622a overexpression significantly inhibits PCa metastasis (Figures $8 \mathrm{c}$ and d).
Overexpression of miR-3622a suppresses proliferation in $\mathrm{PCa}$ cell lines. Cellular proliferation, survival and migration are among the common functions required for tumor progression and metastasis in target microenvironments. In view of the observed effects of miR-3622a expression on progression and metastasis in xenograft mouse models, we also assayed the effects of miR-3622a overexpression on proliferation and survival in PCa cells lines (Supplementary Figure S6). miR-3622a overexpression (Figure 4a) significantly suppressed the proliferation of $\mathrm{PC} / \mathrm{LNCaP} /$ Du145 cells as assessed by clonogenicity assay (Supplementary Figure S6A). A significant decrease in cell viability was observed over time in PC3/ LNCaP/Du145 cells overexpressing miR-3622a as compared to control (Supplementary Figure S6B). Cell cycle analyses in PC3 cells showed that miR-3622a overexpression induces G0-G1 cell cycle arrest $(P=0.0046)$ (Supplementary Figure S6C). This suggests that miR-3622a suppresses tumorigenesis, in part, through its suppressive effects on cellular proliferation. 


\section{Discussion}

Here we demonstrate for the first time that miR-3622a located at chromosome 8p21 is a key negative regulator of PCa EMT. Genetic studies have consistently reported the loss of chr8p $\mathrm{p}^{23-31}$ as a frequent alteration of the PCa oncogenome that has been associated with the loss of prostate-specific NKX3.1 gene. ${ }^{32}$ Our study uncovers the role of a novel alternative tumor suppressor miRNA in this region with crucial role in PCa EMT, tumor progression and metastasis. Though this region has been associated with $\mathrm{PCa}$ initiation, a significantly higher deletion frequency has been reported in advanced $\mathrm{PCa},{ }^{33,35}$ suggesting its role in $\mathrm{PCa}$ progression. Importantly, we provide the first evidence that miR-3622a promotes the epithelial phenotype and inhibits EMT by directly repressing key EMT-TFs, including ZEB1, SNAI2/SLUG and CTNNB1. These TFs are repressors that coordinate the EMT process by repressing E-cadherin and tumor-related genes. ${ }^{43-47}$ Further, our in vivo data confirm the suppressive role of miR-3622a in PCa progression and metastasis.

Our study establishes miR-3622a attenuation as a major $\mathrm{PCa}$ alteration. Analyses of the regulatory mechanisms underlying low miR-3622a expression suggest that apart from genomic deletions of this locus, epigenetic events such as DNA methylation regulate miR-3622a expression. It has been previously demonstrated that miRNAs are inactivated by specific genetic and epigenetic alterations. ${ }^{48-51}$ Further, low miR-3622a expression was found to be associated with poor survival outcome and higher Gleason grade tumors. This finding is in concordance with previous studies that show a significant increase in chr8p deletions with tumor grade, ${ }^{35}$ progression and poor prognosis in $\mathrm{PCa}^{36}$ Our findings suggest that miR-3622a may be useful as a prognostic and diagnostic marker for $\mathrm{PCa}$. Further studies with larger clinical cohorts are warranted to test the prognostic potential of miR-3622a in PCa.

In conclusion, our study suggests that frequent loss of miR-3622a at chr8p21 region by genetic and epigenetic mechanisms lead to EMT induction by upregulation of ZEB1 and SNAI2 that, in turn, promotes $\mathrm{PCa}$ progression and metastasis (Figure 8e). Thus, we have identified an important miRNA component of a frequently lost chromosomal region associated with $\mathrm{PCa}$, which is a highly significant step towards understanding the mechanistic involvement of this locus. Our findings lend support to the concept that CNAs frequently include multiple genes that may cooperate to produce more aggressive disease. ${ }^{52}$ In conclusion, miR-3622a is a potential novel PCa biomarker and may be a novel drug target for developing therapeutic regimens against advanced $\mathrm{PCa}$.

\section{Materials and Methods}

Cell lines and cell culture. Primary prostate epithelial cells (PPEC) and prostate carcinoma cell lines (LNCaP, Du145, PC3) were obtained from the American Type Culture Collection (ATCC) and cultured under recommended conditions. PPEC were maintained in prostate epithelial cell basal media supplemented with prostate epithelial cell growth kit (ATCC) under recommended conditions. LNCaP, PC3 cells were maintained in RPMI 1640 media (UCSF cell culture facility) and Du145 cells were cultured in MEM media, each supplemented with $10 \%$ fetal bovine serum (FBS) (Atlanta biologicals) and $1 \%$ penicillin/ streptomycin (UCSF cell culture facility). Immortalized non-transformed prostate epithelial cell line (BPH1) ${ }^{42}$ was maintained in RPMl 1640 media supplemented with
$5 \%$ FBS, and $1 \%$ penicillin/streptomycin. All cell lines were maintained in an incubator with a humidified atmosphere of $95 \%$ air and $5 \% \mathrm{CO} 2$ at $37^{\circ} \mathrm{C}$. Prostate cell lines were authenticated by DNA short-tandem repeat analysis. The experiments with cell lines were performed within 6 months of their procurement/ resuscitation.

Tissue samples. Formalin-fixed, paraffin-embedded (FFPE) PCa samples were obtained from the SFVAMC. Written informed consent was obtained from all patients and the study was approved by the UCSF Committee on Human Research. All slides were reviewed by a board certified pathologist for the identification of $\mathrm{PCa}$ foci as well as adjacent normal glandular epithelium. Tissues were micro dissected as described in ${ }^{53}$ and detailed in Supplementary Methods.

miRNA/siRNA transfections. Cells were plated in growth medium without antibiotics $\sim 24 \mathrm{~h}$ before transfections. Transient transfections of miRNA precursor (Ambion, Thermo Fisher Scientific Life Sciences, Rockford, IL, USA) /siRNA (Origene, Rockville, MD, USA) was carried out by using Lipofectamine 2000 (Invitrogen, Thermo Fisher Scientific Life Sciences, Rockford, IL, USA) according to the manufacturer's protocol. miR-3622a precursor (AM17101) or negative control (miR-CON) (AM17110) (Ambion) was used for miRNA transfections followed by functional assays. Trilencer-27 predesigned siRNA (Origene) was used for siRNAmediated knockdowns of ZEB1 (SR304746), SNAI2 (SR304468), and CTNNB1 (SR301063). All miRNA/siRNA transfections were for $72 \mathrm{~h}$.

Animal studies. Bioluminescent PC-3M-luc cells stably transfected with control $\mathrm{miR} / \mathrm{miR}-3622 a$ were surgically implanted in the dorsal prostate lobe or injected into the left ventricle of nu/nu mice (Simonsen Laboratories, Gilroy, CA, USA) followed by periodic monitoring of tumor development using bioluminescence detection. All animal care was in accordance with the guidelines of the SFVAMC and the study was approved by the San Francisco VA IACUC.

TCGA data. The level 3 miRNAseq data for prostate adenocarcinomas were downloaded from The Cancer Genome Atlas (TCGA) data portal (https://tcga-data. nci.nih.gov/tcga/dataAccessMatrix.htm). At the time of download, this dataset had the miRNA expression data derived from the Illumina HiSeq platform for a total of 184 prostate tumors. The copy number data, RNAseq data and the clinical data for the prostate adenocarcinomas in the TCGA dataset were retrieved from cBioportal. $^{40,41}$

Statistics. All the quantified data represent an average of triplicate samples or as indicated. The data are represented as mean \pm S.E.M or as indicated. Two-tailed Student's $t$-test was used for comparisons between groups. All statistical analyses were performed using MedCalc version 10.3.2 (Medcalc Software; Acacialaan 22, Ostend, Belgium). Results were considered statistically significant at $P \leqslant 0.05$.

Full method descriptions are available in the Supplementary Methods.

\section{Conflict of Interest}

The authors declare no conflict of interest.

Acknowledgements. We thank Dr. Roger Erickson for his support with preparation of the manuscript. We acknowledge UCSF CTSI (Dr. Iryna V. Lobach) for help with statistical analysis. This work was supported by the National Cancer Institute at the NIH (Grant Number R01CA177984 to SS; R01CA138642 to RD), VA program project (BX001604).

1. Kalluri R, Weinberg RA. The basics of epithelial-mesenchymal transition. J Clin Invest 2009; 119: $1420-1428$.

2. Thiery JP. Epithelial-mesenchymal transitions in tumour progression. Nat Rev Cancer 2002; 2: $442-454$.

3. Zhang J, Ma L. MicroRNA control of epithelial-mesenchymal transition and metastasis. Cancer Metastasis Rev 2012; 31: 653-662.

4. Cheng L, Nagabhushan M, Pretlow TP, Amini SB, Pretlow TG. Expression of E-cadherin in primary and metastatic prostate cancer. Am J Pathol 1996; 148: 1375-1380.

5. Nauseef JT, Henry MD. Epithelial-to-mesenchymal transition in prostate cancer: paradigm or puzzle? Nat Rev Urol 2011; 8: 428-439.

6. Sethi S, Macoska J, Chen W, Sarkar FH. Molecular signature of epithelial-mesenchymal transition (EMT) in human prostate cancer bone metastasis. Am J Translat Res 2010; 3: 90-99. 
7. Wallerand H, Robert G, Pasticier G, Ravaud A, Ballanger P, Reiter RE et al. The epithelialmesenchymal transition-inducing factor TWIST is an attractive target in advanced and/or metastatic bladder and prostate cancers. Urol Oncol 2010; 28: 473-479.

8. Gravdal K, Halvorsen OJ, Haukaas SA, Akslen LA. A switch from E-cadherin to N-cadherin expression indicates epithelial to mesenchymal transition and is of strong and independent importance for the progress of prostate cancer. Clin Cancer Res 2007; 13: 7003-7011.

9. Hansel DE, Epstein JI. Sarcomatoid carcinoma of the prostate: a study of 42 cases. Am J Surg Pathol 2006; 30: 1316-1321.

10. Sekhon K, Bucay N, Majid S, Dahiya R, Saini S. MicroRNAs and epithelial-mesenchymal transition in prostate cancer. Oncotarget 2016; 7: 67597-67611.

11. Bracken CP, Gregory PA, Khew-Goodall Y, Goodall GJ. The role of microRNAs in metastasis and epithelial-mesenchymal transition. Cell Mol Life sci 2009; 66: 1682-1699.

12. Kang $Y$, Massague J. Epithelial-mesenchymal transitions: twist in development and metastasis. Cell 2004; 118: 277-279.

13. Zhang J, Ma L. MicroRNA control of epithelial-mesenchymal transition and metastasis. Cancer Metastasis Rev 2012; 31: 653-662.

14. Bartel DP. MicroRNAs: target recognition and regulatory functions. Cell 2009; 136: 215-233.

15. Gregory PA, Bracken CP, Bert AG, Goodall GJ. MicroRNAs as regulators of epithelialmesenchymal transition. Cell Cycle 2008; 7: 3112-3118.

16. Gregory PA, Bert AG, Paterson EL, Barry SC, Tsykin A, Farshid G et al. The miR-200 family and miR-205 regulate epithelial to mesenchymal transition by targeting ZEB1 and SIP1. Nat Cell Biol 2008; 10: 593-601.

17. Burk U, Schubert J, Wellner U, Schmalhofer O, Vincan E, Spaderna S et al. A reciprocal repression between ZEB1 and members of the miR-200 family promotes EMT and invasion in cancer cells. EMBO Rep 2008; 9: 582-589.

18. Gandellini P, Folini M, Longoni N, Pennati M, Binda M, Colecchia M et al. miR-205 Exerts tumor-suppressive functions in human prostate through down-regulation of protein kinase Cepsilon. Cancer Res 2009; 69: 2287-2295.

19. Korpal M, Kang Y. The emerging role of miR-200 family of microRNAs in epithelialmesenchymal transition and cancer metastasis. RNA Biol 2008; 5: 115-119.

20. Korpal M, Lee ES, Hu G, Kang Y. The miR-200 family inhibits epithelial-mesenchymal transition and cancer cell migration by direct targeting of E-cadherin transcriptional repressors ZEB1 and ZEB2. J Biol Chemy 2008; 283: 14910-14914.

21. Park SM, Gaur AB, Lengyel E, Peter ME. The miR-200 family determines the epithelial phenotype of cancer cells by targeting the E-cadherin repressors ZEB1 and ZEB2. Genes Dev 2008; 22: 894-907.

22. Saini S, Majid S, Yamamura S, Tabatabai L, Suh SO, Shahryari V et al. Regulatory role of mir-203 in prostate cancer progression and metastasis. Clin Cancer Res 2011; 17: $5287-5298$.

23. Kim JH, Dhanasekaran SM, Mehra R, Tomlins SA, Gu W, Yu J et al. Integrative analysis of genomic aberrations associated with prostate cancer progression. Cancer Res 2007; 67: 8229-8239.

24. Lapointe J, Li C, Giacomini CP, Salari K, Huang S, Wang P et al. Genomic profiling reveals alternative genetic pathways of prostate tumorigenesis. Cancer Res 2007; 67: 8504-8510.

25. Lapointe J, Li C, Higgins JP, van de Rijn M, Bair E, Montgomery K et al. Gene expression profiling identifies clinically relevant subtypes of prostate cancer. Proc Natl Acad Sci USA 2004; 101: 811-816.

26. Lieberfarb ME, Lin M, Lechpammer M, Li C, Tanenbaum DM, Febbo PG et al. Genome-wide loss of heterozygosity analysis from laser capture microdissected prostate cancer using single nucleotide polymorphic allele (SNP) arrays and a novel bioinformatics platform dChipSNP. Cancer Res 2003; 63: 4781-4785.

27. Perner S, Demichelis F, Beroukhim R, Schmidt FH, Mosquera JM, Setlur S et al. TMPRSS2: ERG fusion-associated deletions provide insight into the heterogeneity of prostate cancer. Cancer Res 2006; 66: 8337-8341.

28. Singh D, Febbo PG, Ross K, Jackson DG, Manola J, Ladd C et al. Gene expression correlates of clinical prostate cancer behavior. Cancer Cell 2002; 1: 203-209.

29. Taylor BS, Schultz N, Hieronymus H, Gopalan A, Xiao Y, Carver BS et al. Integrative genomic profiling of human prostate cancer. Cancer Cell 2010; 18: 11-22.

30. Emmert-Buck MR, Vocke CD, Pozzatti RO, Duray PH, Jennings SB, Florence CD et al. Allelic loss on chromosome 8p12-21 in microdissected prostatic intraepithelial neoplasia. Cancer Res 1995; 55: 2959-2962.

31. Vocke CD, Pozzatti RO, Bostwick DG, Florence CD, Jennings SB, Strup SE et al. Analysis of 99 microdissected prostate carcinomas reveals a high frequency of allelic loss on chromosome 8p12-21. Cancer Res 1996; 56: 2411-2416.
32. He WW, Sciavolino PJ, Wing J, Augustus M, Hudson P, Meissner PS et al. A novel human prostate-specific, androgen-regulated homeobox gene (NKX3.1) that maps to 8p21, a region frequently deleted in prostate cancer. Genomics 1997; 43: 69-77.

33. Oba K, Matsuyama H, Yoshihiro S, Kishi F, Takahashi M, Tsukamoto M et al. Two putative tumor suppressor genes on chromosome arm $8 \mathrm{p}$ may play different roles in prostate cancer. Cancer Genet Cytogenet 2001; 124: 20-26.

34. Williams JL, Greer PA, Squire JA. Recurrent copy number alterations in prostate cancer: an in silico meta-analysis of publicly available genomic data. Cancer Genet 2014; 207: 474-488.

35. Matsuyama H, Pan Y, Yoshihiro S, Kudren D, Naito K, Bergerheim US et al. Clinical significance of chromosome 8p, 10q, and 16q deletions in prostate cancer. Prostate 2003; 54: 103-111.

36. El Gammal AT, Bruchmann M, Zustin J, Isbarn H, Hellwinkel OJ, Kollermann $\mathrm{J}$ et al. Chromosome $8 p$ deletions and $8 q$ gains are associated with tumor progression and poor prognosis in prostate cancer. Clin Cancer Res 2010; 16: 56-64.

37. Bucay N, Sekhon K, Majid S, Yamamura S, Shahryari V, Tabatabai ZL et al. Novel tumor suppressor microRNA at frequently deleted chromosomal region 8 p21 regulates epidermal growth factor receptor in prostate cancer. Oncotarget 2016; 7: 70388-70403.

38. Persson H, Kvist A, Rego N, Staaf J, Vallon-Christersson J, Luts L et al. Identification of new microRNAs in paired normal and tumor breast tissue suggests a dual role for the ERBB2/ Her2 gene. Cancer Res 2011; 71: 78-86.

39. Witten D, Tibshirani R, Gu SG, Fire A, Lui WO. Ultra-high throughput sequencing-based small RNA discovery and discrete statistical biomarker analysis in a collection of cervical tumours and matched controls. BMC Biol 2010; 8: 58

40. Cerami E, Gao J, Dogrusoz U, Gross BE, Sumer SO, Aksoy BA et al. The cBio cancer genomics portal: an open platform for exploring multidimensional cancer genomics data. Cancer Discov 2012; 2: 401-404.

41. Gao J, Aksoy BA, Dogrusoz U, Dresdner G, Gross B, Sumer SO et al. Integrative analysis of complex cancer genomics and clinical profiles using the cBioPortal. Sci Signal 2013; 6: pl1.

42. Hayward SW, Dahiya R, Cunha GR, Bartek J, Deshpande N, Narayan P. Establishment and characterization of an immortalized but non-transformed human prostate epithelial cell line: BPH-1. In vitro Cell Dev Biol Anim 1995; 31: 14-24.

43. Imamichi $\mathrm{Y}$, Konig A, Gress T, Menke A. Collagen type I-induced Smad-interacting protein 1 expression downregulates E-cadherin in pancreatic cancer. Oncogene 2007; 26: 2381-2385.

44. Spaderna S, Schmalhofer O, Hlubek F, Berx G, Eger A, Merkel S et al. A transient, EMTlinked loss of basement membranes indicates metastasis and poor survival in colorectal cancer. Gastroenterology 2006; 131: 830-840.

45. Spaderna S, Schmalhofer O, Wahlbuhl M, Dimmler A, Bauer K, Sultan A et al. The transcriptional rpressor ZEB1 promotes metastasis and loss of cell polarity in cancer. Cancer Res 2008; 68: 537-544.

46. Spoelstra NS, Manning NG, Higashi Y, Darling D, Singh M, Shroyer KR et al. The transcription factor ZEB1 is aberrantly expressed in aggressive uterine cancers. Cancer Res 2006; 66: 3893-3902.

47. Comijn J, Berx G, Vermassen P, Verschueren K, van Grunsven L, Bruyneel E et al. The two-handed $\mathrm{E}$ box binding zinc finger protein SIP1 downregulates $\mathrm{E}$-cadherin and induces invasion. Mol Cell 2001; 7: 1267-1278.

48. Bueno MJ, Perez de Castro I, Gomez de Cedron M, Santos J, Calin GA, Cigudosa JC et al. Genetic and epigenetic silencing of microRNA-203 enhances ABL1 and BCR-ABL1 oncogene expression. Cancer Cell 2008; 13: 496-506.

49. Calin GA, Dumitru CD, Shimizu M, Bichi R, Zupo S, Noch E et al. Frequent deletions and down-regulation of micro- RNA genes miR15 and miR16 at 13q14 in chronic lymphocytic leukemia. Proc Natl Acad Sci US A 2002; 99: 15524-15529.

50. Varambally S, Cao Q, Mani RS, Shankar S, Wang X, Ateeq B et al. Genomic loss of microRNA-101 leads to overexpression of histone methyltransferase EZH2 in cancer. Science 2008; 322: 1695-1699.

51. Zhang L, Huang J, Yang N, Greshock J, Megraw MS, Giannakakis A et al. microRNAs exhibit high frequency genomic alterations in human cancer. Proc Natl Acad Sci USA 2006; 103: 9136-9141.

52. Xue W, Kitzing T, Roessler S, Zuber J, Krasnitz A, Schultz N et al. A cluster of cooperating tumor-suppressor gene candidates in chromosomal deletions. Proc Natl Acad Sci USA 2012; 109: 8212-8217.

53. Bucay N, Shahryari V, Majid S, Yamamura S, Mitsui Y, Tabatabai ZL et al. miRNA expression analyses in prostate cancer clinical tissues. J Vis Exp 2015 (doi:10.3791/53123).

Supplementary Information accompanies this paper on Cell Death and Differentiation website (http://www.nature.com/cdd) 